\title{
Sustained pharyngeal inflation on upper airway effects in infants - flexible bronchoscopy measurement
}

\author{
Wen-Jue Soong ${ }^{1}$, Chia-Feng Yang ${ }^{2}$, Chieh-Ho Chen ${ }^{3}$, Yu-Sheng Lee ${ }^{4}$, Chien-Heng Lin ${ }^{5}$, \\ Pei-Chen Tsao ${ }^{6}$, and Nitin Dhochak ${ }^{7}$ \\ ${ }^{1}$ Children;s Hospital, Taichung China Medical University \\ ${ }^{2}$ Taipei veterans general hospital \\ ${ }^{3}$ China Medical University \\ ${ }^{4}$ Taipei Veterans General Hospital \\ ${ }^{5}$ China Medical University Hospital \\ ${ }^{6}$ Veteran general hospital, Taipei, Taiwan (ROC) \\ ${ }^{7}$ All India Institute of Medical Sciences
}

March 18, 2021

\begin{abstract}
OBJECTIVE: Sustained pharyngeal inflation (SPI) with pharyngeal oxygen and nose-close (PhO2-NC) can create positive peak inflation pressure (PIP) inside the pharyngolaryngeal space (PLS). This study measured and compared effects of four different SPI durations in the PLS. METHOD: In this prospective observational study, 20 consecutive infants aged less than 3 years, scheduled for elective flexible-bronchoscopy were enrolled. SPI was performed twice in four different durations $(0,1,3$ and 5 seconds) sequentially in each infant. PIP was measured for each SPI in the pharynx, and simultaneously took images at two locations of oropharynx and supra-larynx. Infants' demographic details and PIP levels, lumen expansion scores and images of PLS were measured and analyzed. RESULTS: Twenty infants with 40 measurements were collected. The mean (SD) age and weight were 11.6 (9.1) months and $6.8(2.4) \mathrm{kg}$, respectively. The measured mean (SD) pharyngeal PIPs were 4.1 (3.3), $21.9(7.0), 42.2(12.3)$ and $65.5(18.5) \mathrm{cm} \mathrm{H} 2 \mathrm{O}$ at SPI duration of $0,1,3$ and 5 seconds, respectively; which showed significant positive association $(\mathrm{p}<0.001)$. At assigned locations, the corresponding PLS images also show significant increase in lumen expansion scores and number of detected lesions with increase in SPI duration $(\mathrm{p}<0.001)$. The mean (SD) study time was 5.7 (1.2) minutes. No study related complication was noted. CONCLUSIONS: SPI with PhO2-NC up to 5 seconds is a simple, safe and feasible clinical ventilation modality. It may provide enough PIP to expand the PLS and facilitate flexible-bronchoscopy performance in infants.
\end{abstract}

Title pages

Sustained pharyngeal inflation on upper airway effects in infants - flexible bronchoscopy measurement

Wen-Jue Soong ${ }^{1,2,3}$, Chia-Fang Yang ${ }^{2,3,4}$, Yu-Sheng Lee ${ }^{2,3,4}$, Pei-Jeng Tsao ${ }^{2,3,4}$, Chien-Heng Lin ${ }^{1}$, Chieh-Ho Chen ${ }^{1}$, Nitin Dhochak ${ }^{5}$

${ }^{1}$ Division of Pediatric Pulmonology, Children's Hospital, China Medical University, Taichung, Taiwa

${ }^{2}$ Department of Pediatrics, Taipei Veterans General Hospital, Taipei, Taiwan

${ }^{3}$ Department of Pediatrics, Triservice General Hospital, National Defence Medical Ceter, Taipei, Taiwan;

${ }^{4}$ School of Medicine, National Yang-Ming University; Taipei, Taiwan 
${ }^{5}$ Department of Pediatrics, All India Institute of Medical Sciences, New Delhi, India

Key words :

Flexible bronchoscopy, noninvasive ventilation, pharyngeal pressure, Soong's ventilation, sustained inflation Corresponding (reprint request) author:

\section{Wen-Jue Soong, MD}

Division of Pediatric Pulmonology, China Medical University Children's Hospital, Taichung, Taiwan.

No. 2, Yuh-Der Road, Taichung 40447, Taiwan.

Phone: +886-4-22052121 ext. 4648;

Fax.: +886-4-22032798;

Cell phone: +886-921067989;

E-mail: wjsoong3@gmail.com

\section{Financial disclosure:}

- This study was granting supported and IRB proved by both two organizations of: 1) Taipei-Veterans General Hospital (TVGH no.: V107C-179) and 2) China Medical University Children's Hospital (DMR109-179).

- There is no support from any manufacture or company about this study.

- The first author, Wen-Jue Soong, wrote the first draft of the manuscript.

- Report to Pediatric Pulmonology: on behalf of all authors, the corresponding author states that there is no conflict of interest with any person or company.

\section{Authors list}

\begin{tabular}{llll}
\hline Name & $\begin{array}{l}\text { Highest academic } \\
\text { degree }\end{array}$ & E-mail & Affiliation \\
\hline Wen-Jue Soong & MD. & wjsoong3@gmail.com & Division of Pediatric \\
& & Pulmonology Children's \\
& & Hospital, China Medical \\
& & University Taichung, \\
& & Taiwan. Department of \\
& & Pediatrics, Taipei \\
& & Veterans General \\
& & Hospital, Taipei; \\
& & Department of Pediatrics, \\
& & Triservice General \\
& & Hospital, National \\
& & Defence Medical Ceter, \\
& & Taipei, Taiwan; School of \\
Chia-Feng Yang & PhD & Medicine, National \\
Yu-Sheng Lee & PhD & Yang-Ming University; \\
Pei-Chen Tsao & PHD & Taipei, Taiwan \\
& & Cfyang3@vghtpe.gov.tw & \\
& & leeys@vghtpe.gov.tw &
\end{tabular}




\begin{tabular}{llll}
\hline Name & $\begin{array}{l}\text { Highest academic } \\
\text { degree }\end{array}$ & E-mail & Affiliation \\
\hline Chieh-Ho Chen & MD & pedlungcmu@gmail.com & $\begin{array}{l}\text { 1.Children's Hospital, } \\
\text { China Medical } \\
\text { University, Taichung, } \\
\text { Taiwan }\end{array}$ \\
Chien-Heng Lin & MD & & Lch227@ms39.hinet.net \\
Nitin Dhochak & MD. DM. & nitind.aiims@gmail.com & 5.Department of \\
& & Pediatrics, All India \\
& & Institute of Medical \\
& & Sciences, New Delhi, \\
& & India \\
& & Current post: Research \\
& & Officer \\
\hline
\end{tabular}

Abbreviated title: Sustained pharyngeal inflation effects in upper airway

Abstract

OBJECTIVE: Sustained pharyngeal inflation (SPI) with pharyngeal oxygen and nose-close $\left(\mathrm{PhO}_{2}-\mathrm{NC}\right)$ can create positive peak inflation pressure (PIP) inside the pharyngolaryngeal space (PLS). This study measured and compared effects of four different SPI durations in the PLS.

METHOD: In this prospective observational study, 20 consecutive infants aged less than 3 years, scheduled for elective flexible-bronchoscopy were enrolled. SPI was performed twice in four different durations $(0,1,3$ and 5 seconds) sequentially in each infant. PIP was measured for each SPI in the pharynx, and simultaneously took images at two locations of oropharynx and supra-larynx. Infants' demographic details and PIP levels, lumen expansion scores and images of PLS were measured and analyzed.

RESULTS: Twenty infants with 40 measurements were collected. The mean (SD) age and weight were 11.6 (9.1) months and $6.8(2.4) \mathrm{kg}$, respectively. The measured mean (SD) pharyngeal PIPs were 4.1 (3.3), 21.9 (7.0), $42.2(12.3)$ and $65.5(18.5) \mathrm{cm} \mathrm{H}_{2} \mathrm{O}$ at SPI duration of $0,1,3$ and 5 seconds, respectively; which showed significant positive association $(\mathrm{p}<0.001)$. At assigned locations, the corresponding PLS images also show significant increase in lumen expansion scores and number of detected lesions with increase in SPI duration $(\mathrm{p}<0.001)$. The mean $(\mathrm{SD})$ study time was $5.7(1.2)$ minutes. No study related complication was noted.

CONCLUSIONS: SPI with $\mathrm{PhO}_{2}$-NC up to 5 seconds is a simple, safe and feasible clinical ventilation modality. It may provide enough PIP to expand the PLS and facilitate flexible-bronchoscopy performance in infants.

\section{Introduction}

Sustained pharyngeal inflation (SPI) is an assist positive pressure ventilation (PPV) maneuver with prolonged inspiration time while a positive and a peak inflation pressure (PIP) can be created inside the pharyngolaryngeal space (PLS). In children with a patent airway, the prolonged pressurized-air flow may actively move from the upper into the lower airways and providing the ample time and pressure gradient needed for expanding the airway lumens, alveoli and promoting gas exchange across the air-fluid interface. Traditionally, this sustained inflation is accomplished with a gas flow delivered via different devices of Ambu-facemask, bi-nasal prongs, laryngeal mask airway (LMA), or endotracheal tube (ETT). ${ }^{1-8}$ The sustained inflation has gradually become a widespread clinical practice.

"Pharyngeal oxygen with optional nose-close and abdomen-compression $\left(\mathrm{PhO}_{2}\right.$-NC-AC)" is a novel model of noninvasive ventilation (NIV), without using any artificial device such as Ambu-bag, face or nasal mask, 
LMA, ETT, or mechanical ventilator. It has already been demonstrated to provide adequate oxygenation and ventilation to assist interventional flexible bronchoscopy (FB) in pediatric patients, even in hypoxemic children on respiratory support. ${ }^{9-15} \mathrm{~A}$ prolonged duration of the action of NC indeed mimic an SPI. Theoretically, in a relatively close space like the PLS, with the setting of a continuous $\mathrm{PhO}_{2}$ flow, the ventilation effect of the SPI may create as well as increase the PIP in the PLS. Closely measuring and monitoring the level of these PIPs are clinical imperative. To our knowledge, there is no study dealing with the SPI durations and associated changes of the PIP level and image in the upper airway of PLS in pediatrics.

In this study, we use the "PhO $\mathrm{O}_{2}-\mathrm{NC}$ " as a SPI technique to evaluate and compare the effect of different SPI durations on the associated changes of 1) the PIP levels generated, 2) the airway lumen images, and 3) the lesion detected which measured by FB in the PLS.

\section{Methods}

In a one-year prospective study, 20 consequent infants who full-filled criteria including a) age less than 3 years, b) a clinical schedule for elective FB, and c) got parents signed-consent for this study, were enrolled. Two bronchoscopists (Soong and Chen) carried out the SPI studies. The Institutional Review Board of hospital and IRB approved this study (DMR-109-179, CMUH108-REC3-114). Patient confidentiality was maintained in accordance with Health Insurance Portability and Accountability Act guidelines.

The study child was laid supine on the endoscopy room table, with procedural sedation and continuous cardiopulmonary monitoring. A continuous oxygen flow $(1.0 \mathrm{~L} / \mathrm{Kg} / \mathrm{min}$, maximal $10.0 \mathrm{~L} / \mathrm{min})$ was delivered via a small nasopharyngeal catheter via (right) nostril as the " $\mathrm{PhO}_{2}$ ". This $\mathrm{NIV}$ of $\mathrm{PhO}_{2}-\mathrm{NC}-\mathrm{AC}$ supported the whole FB intervention. All the SPI studies were performed after completion of their original scheduled FB.

\section{PIP measurement}

Figure 1a shows the configuration of this study. A short $(25 \mathrm{~cm})$ working-length FB (OD 3.8mm, HYF-V, Olympus), with an inner channel (1.2 mm), inserted via one side (left) nostril. A small pressure transmitting catheter linked the working channel to a real-time pressure monitor (GB30, Galemed, Taiwan). Therefore, the real-time dynamic intra-pharyngeal pressures could be immediately revealed when the FB tip was freely located in the PLS. The PIP was measured at the end of SPI.

\section{Modes of SPI}

SPI (NC, inspiration) processed in four modes of different duration (0,1,3 and 5 seconds) consecutively, as depicted in Figure 1b. Each SPI maneuver was performed twice at same duration, then processed to the next mode. Each SPI was followed by an open-nose (deflation) period of 10 seconds for expiration and spontaneous breathing. PIP reading was measured and recorded levels for these maneuvers in the PLS.

\section{Lumen imaging}

With the FB visualization in the PLS, series of lumen images were taken along the course of dynamic changes with the inflation. In addition, associated anomaly or lesion if existed could also be detected along the different inflation courses of SPI. Simultaneously, took the lesion image at each plateau of PIP, at two assigned locations of oropharynx, and supra-larynx. All FB images and videos of SPI studies were stored.

For objective evaluation of the lumen changes, the four final captured lumen images of oropharynx and supra-larynx (including the lesions) each at different SPI mode were grouped and sent for evaluation. Their lumen expansion scores were judged independently on a five-point Likert scale (1 to 5: severe collapsed, collapsed, average, expanded, much expanded) within one week by five qualified pediatric pulmonologists who blinded to the sources of these image. The final scores were averaged and analyzed.

Data of patient demographics, PIP levels, expansion scores and images in the PLS were all collected for further analysis.

\section{Statistics}


Analyses for the PIP levels and airway lumen scores were performed using One-way ANOVA of the MedCalc statistical software (version 19.3.1, MedCalc Software Ltd, Acacialaan 22, 8400 Ostend, Belgium). Analyses for the numbers of detected lesion were done using chi-square test and post hoc Bonferroni correction. Statistical differences were considered significant at $p<0.05$.

\section{Results}

In these 20 studied infants (Table 1), the mean (SD) age was 11.6 (9.1) months and the mean (SD) body weight was $6.8(2.4) \mathrm{kg}$. There were 40 sets of measured data. The mean (SD) operative time of this SPI study was 5.7 (1.2) minutes.

Table 2 presents the details of the intrapharyngeal PIP, lumen expansion scores, locations and number of detected lesions on these four SPI modes. In the collected 40 sets of measurements, the mean (SD) PIP in the pharynx were $4.1(3.3), 21.9(7.0), 42.2(12.3)$ and $65.5(18.5) \mathrm{cmH}_{2} \mathrm{O}$ at SPI duration of $0,1,3$ and 5 seconds, respectively. As shows in the Figure 2 , there is positive and significant $(\mathrm{p}<0.001)$ linear increase in PIP levels with SPI duration from 0 through 5 seconds. In these changes of image sequences, there was progressive space expansion and significant greater lumen expansion scores $(p<0.001)$ in all locations of the PLS.

Those pressure-sensitive dynamical lesions, such as pharyngomalacia, laryngeal malacia, laryngeal cleft, glottic malacia, etc., which might be overlooked with a low PIP level of short duration SPI. But after adequate lumen expansion with a high PIP level, definite diagnosis could be achieved. Significantly higher number of lesions could be detected with higher PIP levels $(\mathrm{p}=0.004)$. With the SPI of 5 seconds, the highest PIP, and lumen expansion score were achieved, and FB identified the most number of lesion $(\mathrm{p}<0.001)$.

The corresponding levels of PIP, expansion scores, images of each SPI mode of pharyngeal and laryngeal lesion were illustrated in the Figure 3 and Figure 4, respectively. On the same row, all four images were taken at similar location of same infant but with different PIP levels generated by different SPI durations. In these changes of image sequences, there was a progressive and significant space expansion and greater lumen dimension scores in all locations of the PLS, especially with existing airway pathologic lesions such as pharyngomalacia, uvular cyst, vallecular cyst, etc. During FB assessment, these created lumen expansions also facilitated accurate and comprehensive evaluation of lesions which were not visible in the low PIP levels but became apparent with the high PIP levels of longer SPI duration.

All these SPI studies were successfully completed. There was no any study-related complication such as upper airway bleeding, subcutaneous emphysema, pneumothorax, desaturation $(<90 \%)$ or bradycardia $(<100$ beat/min).

\section{Discussion}

Our study demonstrated significant increase in PIP with the use of simple NIV of "PhO $2-\mathrm{NC}$ " as an SPI technique. To the best of our knowledge, this is the first such study in the medical literature. Even with the frequent use of NIV worldwide, the types of device and the modes of delivery being used vary among countries and institutes. Clinically, this SPI is unique as it waives using other supplementary instruments, except a small oxygen catheter, and bronchoscopist himself can easily handle and control the whole procedure.

The PLS is a fibromuscular tube-like structure that is semicircular in cross section and relatively closed space. It serves as a continuation of the aerodigestive cavity, providing pathway from the nasal and oral cavities to the trachea, esophagus and the bilateral middle ears. PLS boundaries almost with soft tissues of the mouth and the nasal choanae anteriorly; the soft palate, velum, and portion of the skull base superiorly; the tonsils laterally; the inlets of larynx and esophagus inferiorly; and the pharyngeal constrictors posteriorly. Therefore, the PLS is a potential dynamic space which expands with increasing inside pressure.

FB examination via nasal track can offer real-time visualization and assessment of the whole PLS. FB is useful for detecting dynamic images that better demonstrate the presence, location, degree and extent of lesions. In this study, an optional action of SPI by $\mathrm{PhO}_{2}-\mathrm{NC}$ could create enough and controllable 
positive distending pressure which helps to open and expand the PLS, increase the cross-sectional area, and therefore allow accurate and precise inspection the anatomic structure with FB. Especially when there are pressure dynamic lesions which may lead to collapse or block airway path such as pharyngomalacia, adenoid hypertrophy, tonsil hypertrophy, vallecular cyst, laryngomalacia or glottis malacia laryngeal cleft, etc. as shown in the Figure 3 and Figure 4. While patients are sedated, these lesions may cause difficult breathing, hypoxia and desaturation. During FB interventions, these lesions may remain unrecognized when using traditional technique without appropriate or controllable pressure in the PLS. The $\mathrm{PhO}_{2}-\mathrm{NC}$ itself can provide simultaneous and optional PPV. This is not only safe for the patient, but also assists for the therapeutic intervention of FB such as doing laser partial adenoidectomy and tonsillectomy, check bleeding, marsupialization of vallecular cyst, ${ }^{11}$ epiglottoplasty, ${ }^{16}$ etc. Our team has already been using this SPI modality successfully in many pediatric endoscopy interventions of airway ${ }^{9-16}$ and esophagus. ${ }^{17,18}$

Physiologically, this $\mathrm{PhO}_{2}-\mathrm{NC}$ has combinational effects of "apnea oxygenation" ${ }^{19-23}$ and PPV. In the setting of $\mathrm{PhO}_{2}$, the continuous flow indeed fully fills the PLS with oxygen. For apnea oxygenation, randomized controlled trials have shown that it can extend the period of safe apnea, and reduce the unintended hypoxemia when used in patients after sedation or muscle paralysis, even with difficult airways. $\mathrm{PhO}_{2}$ with addition of optional NC as SPI, both PIP and PPV can be created and sustained for a demand period. This allows appropriate and controllable levels of pressure and times to expand the collapsed PLS for FB detail inspection, as well as performing diagnostic and therapeutic interventions. Furthermore, it may also promote the pressurized oxygen flow into the lower airways and lungs. ${ }^{20-24}$

The modality of FB with this SPI offers several clinical advantages. First, transnasal approach of FB provides a good assessment for dynamic movements of airway lumens, as in small infants. Second, the ventilation interface of this SPI is in the PLS, near the larynx, which much reduces the respiratory dead-space. Third, this SPI is an easy PPV technique that can be performed with only a continuous gas (oxygen or air) flow and a soft catheter with distal tip sitting in the pharynx that minimizes impediment to airway access during FB procedures. FB operator can provide PPV himself while simultaneously execute FB interventions. Fourth, SPI can gradually and appropriately open and expand a collapsed PLS, as demonstrated in this study, which facilitates the passage of FB for thorough measurement of the PLS. This allows clear visualization of contents, structures and lesions that helps to yield more accurate diagnosis and subsequent effective management. All of these can be achieved without interference by artificial airway devices of facemask, nasal prong, LMA or ETT. Fifth, it is technically simple and readily available, therefore, can be used in resource-limited scenarios. Sixth, it causes less distortion in PLS structure, less invasive, better-tolerated and cost-effective compared to the traditional techniques of PPV. In this study, the trend of long SPI presents more effective than short SPI to more expansion of airway space for better and detailed measurement which not easily be achieved in lower inflation levels. Furthermore, these positive and dynamic pressures of SPI may also transmit and facilitate tracheobronchial lumen expansion and lung ventilation. For patients with risk of compromised airway and oxygen desaturation, SPI may provide ventilation and reduce hypoxia, therefore, facilitate patient safety and FB procedures.

We observed that significant number of observations achieved very high PIP at SPI duration of 5 seconds. We did not observe any adverse effects of high PIP as it was used only transiently for our study. But safety of such high pressure cannot be confirmed due to small sample size of our cohort. We recommend SPI duration of 1-3 seconds for routine ventilation during FB and procedures, and 5 seconds inflation should be reserved for special situations where lesions are not adequately visible at lower inflation duration.

Some limitations may exist in this study. There might be variation of the tightness of the nose (mouth)closure between, or even, in the same operators. This indeed may affect the level of measured PIP. A more prolonger duration of SPI, up to 15 seconds or more has been reported, ${ }^{3,8}$ but we restricted duration of SPI to 5 seconds as longer duration may create risky high PIP. In clinical practice, the real effort of SPI may be individualized which can be controlled by the operators.

Nonetheless, the findings of the present study are important and suggest the need for further investigations. The opening pressure of a collapse PLS or occult lesions can be measured and which helps to determine 
appropriate pressure of NIV support. The similar SPI effects may also be noted in the lower airway lumens, ventilation, and lung recruitment.

\section{Conclusion:}

We propose that the application of SPI by $\mathrm{PhO}_{2}-\mathrm{NC}$ with different durations is a simple, safe and viable modality. This prolonged, up to five seconds, SPI could provide enough PIP to expand the PLS, benefit FB performance and comprehensive inspection in infants. This technique could unmask otherwise difficult recognized lesions. Further larger controlled trials are needed before widespread clinical application.

\section{Acknowledgements}

None.

\section{Conflict of Interests}

All authors declare that there is no conflict of interests regarding the publication of this paper.

\section{Reference s}

1. Lista G. · La Verde P.A. Castoldi F. Sustained Inflation and Its Role in the Delivery Room Management of Preterm Infants. Neonatology 2016;109:366-368. https://doi.org/10.1159/000444899

2. Cardiopulmonary resuscitation with chest compressions during sustained inflations a new technique of neonatal resuscitation that improves recovery and survival in a neonatal porcine model. Circulation. 2013;128:2495-2503.

3. Fischer HS, Schmölzer GM, Po-Yin Cheung PY, Bührer C. Sustained inflations and avoiding mechanical ventilation to prevent death or bronchopulmonary dysplasia: a meta-analysis. Eur Respir Rev 2018;27: 180083 [https://doi.org/10.1183/16000617.0083-2018].

4. van Vonderen JJ, Hooper SB, Hummler HD, Lopriore E, te Pas AB: Effects of a sustained inflation in preterm infants at birth. J Pediatr 2014;165:903-908.e1.

5. Walsh MC, Carlo WA. Sustained inflation during HFOV improves pulmonary mechanics and oxygenation. J Appl Physiol. 1988;65:368-72. doi:10.1152/jappl.1988.65.1.368.

6. Keszler M. Sustained inflation during neonatal resuscitation. Curr Opin Pediatr. 2015;27:145-51. doi: 10.1097/MOP.0000000000000204.

7. Schmölzer GM, Kumar M, Aziz K, et al. Sustained inflation versus positive pressure ventilation at birth: a systematic review and meta-analysis. Arch Dis Child Fetal Neonatal. 2015;100:F361-8. doi:10.1136/archdischild-2014-306836. Epub 2014 Dec 30.

8. Chao KY, Lin YW, Chiang CE, Tseng CW, Mu SC. Sustained inflation: The lung recruitment maneuvers for neonates. Paediatr Respir Rev. 2019;21;S1526-0542(19)30095-8. doi: 10.1016/j.prrv.2019.09.010.

9. Soong WJ, Jeng MJ, Lee YS, Tsao PC, Harloff M, Matthew Soong YH. A novel technique of noninvasive ventilation: Pharyngeal oxygen with nose-closure and abdominal-compression- Aid for pediatric flexible bronchoscopy. Pediatr Pulmonol. 2015;50:568-575.

10. Peng YY, Soong WJ, Lee YS, Tsao PC, Yang CF, Jeng MJ. Flexible bronchoscopy as a valuable diagnostic and therapeutic tool in pediatric intensive care patients: a report on 5 years of experience. Pediatr Pulmonol. 2011;46:1031-7. doi:10.1002/ppul.21464. Epub 2011 May 27.

11. Soong WJ, Yang CF, Lee YS, Tso PJ, Lin CH, Chen CH. Vallecular cyst with coexisting laryngomalacia: successful diagnosis and laser therapy by flexible endoscopy with a novel noninvasive ventilation support in infants. Ped Pulmonol 2020:1-7. DOI: 10.1002/ppul.24796

12. Soong WJ, Pediatric Interventional Flexible Bronchoscopy. Pediatr Respiro Crit Care Med. 2018; 2: 38-44. http://www.prccm.org on Monday, October 29, 2018, IP: 203.64.247.4

13. Soong WJ, Tsao PC, Lee YS, Yang CF. Flexible endoscopy for pediatric tracheobronchial metallic stent placement, maintenance and long-term outcomes. PLoS ONE 2018:13: e0192557. https://doi.org/10.1371/journal. pone.0192557 
14. Soong WJ, Tsao PC, Lee YS, Yang CF. Therapeutic flexible airway endoscopy of small children in a tertiary referral center-11 years' experience. PLoS ONE 2017:12(8): e0183078. https://doi.org/10.1371/journal.pone.0183078

15. Soong WJ, Lee YS, Tsao PC, Yang CF. Retrieval of Tracheobronchial foreign bodies by short flexible endoscopy in children. Int J Pediatr Otorhinolaryngol. 2017:95;109-113.

16. Soong WJ, Shiao AS, Jeng MJ, Lee YS, Tsao PC, Yang CF, Soong YH. Comparison between rigid and flexible laser supraglottoplasty in the treatment of severe laryngomalacia in infants. Int J Pediatr Otorhinolaryngol. 2011;75(6):824-9. doi: 10.1016/j.ijporl.2011.03.016. Epub 2011 Apr 21.

17. Hsieh KH, Soong WJ, Jeng MJ, Lee YS, Tsao PC, Chou YL. Flexible endoscopic diagnosis and treatment of esophageal stenosis in children with noninvasive ventilation support. Pediatr Neonatol. 2018:59:31-34. pii: S1875-9572(17)30288-7. doi: 10.1016/j.pedneo.2016.11.003

18. Choua CS, Soong WJ, Lee YS, Tsaoa PC, Chen WY, Chen SJ, et al. Fiberoptic bronchoesophagoscopyassisted evaluation and prognostic factor analysis in children with congenital esophageal atresia and tracheoesophageal fistula. J Chin Med Assoc. 2020;83:180-187. doi: 10.1097/JCMA.0000000000000246.

19. Soneru CN, Hurt HF, Petersen TR, Davis DD, Braude DA, Falcon RJ. Apneic nasal oxygenation and safe apnea time during pediatric intubations by learners. Paediatr Anaesth. 2019;29:628-634. https://doi.org/10.1111/pan.13645

20. Overmann KM, Boyd SD, Zhang Y, Kerrey BT. Apneic oxygenation to prevent oxyhemoglobin desaturation during rapid sequence intubation in a pediatric emergency department. Am J Emerg Med. 2019;37:1416-1421.

21. Riva T, Seiler S, Stucki F, Greif R, Theiler L. High-flow nasal cannula therapy and apnea time in laryngeal surgery. Paediatr Anaesth. 2016;26:1206-1208.

22. Windpassinger M, Plattner O, Gemeiner J, et al. Pharyngeal oxygen insufflation during airtraq laryngoscopy slows arterial desaturation in infants and small children. Anesth Analg. 2016;122: 1153-1157.

23. Steiner JW, Sessler DI, Makarova N, et al. Use of deep laryngeal oxygen insufflation during laryngoscopy in children: a randomized clinical trial. Br J Anaesth. 2016;117:350-357.

24. Soong WJ, Jeng MJ, Lee YS, Tsao PC, Soong YH. Nasopharyngeal oxygen with intermittent noseclose and abdomen-compression: a novel resuscitation technique in a piglet model. Pediatr Pulmonol. 2013;48:288-294. doi: 10.1002/ppul.22592.

Figure legends

Figure 1. Flexible endoscopy with nasopharyngeal oxygen support: a) nose-open with narrow pharyngeal space; b) SPI (nose-close) expands the pharyngeal space for clear inspection of the structures.

Figure 2. The trend of measured intra-pharyngeal peak pressure with different durations of sustained pharyngeal inflation.

Figure 3. Changes of intra-pharyngeal space (IPS) peak inflation pressure (PIP), lumen dimension score and images ${ }^{\#}$ of pharyngeal lesions in four durations of sustained pharyngeal inflation (SPI).

Figure 4. Changes of intra-pharyngeal space (IPS) peak inflation pressure (PIP), lumen dimension score and images $\#$ of laryngeal lesions in four durations of sustained pharyngeal inflation (SPI).

\section{Hosted file}

\#Table-1.pdf available at https://authorea.com/users/370890/articles/514298-sustainedpharyngeal-inflation-on-upper-airway-effects-in-infants-flexible-bronchoscopymeasurement

\section{Hosted file}

\#Table-2.pdf available at https://authorea.com/users/370890/articles/514298-sustainedpharyngeal-inflation-on-upper-airway-effects-in-infants-flexible-bronchoscopymeasurement 

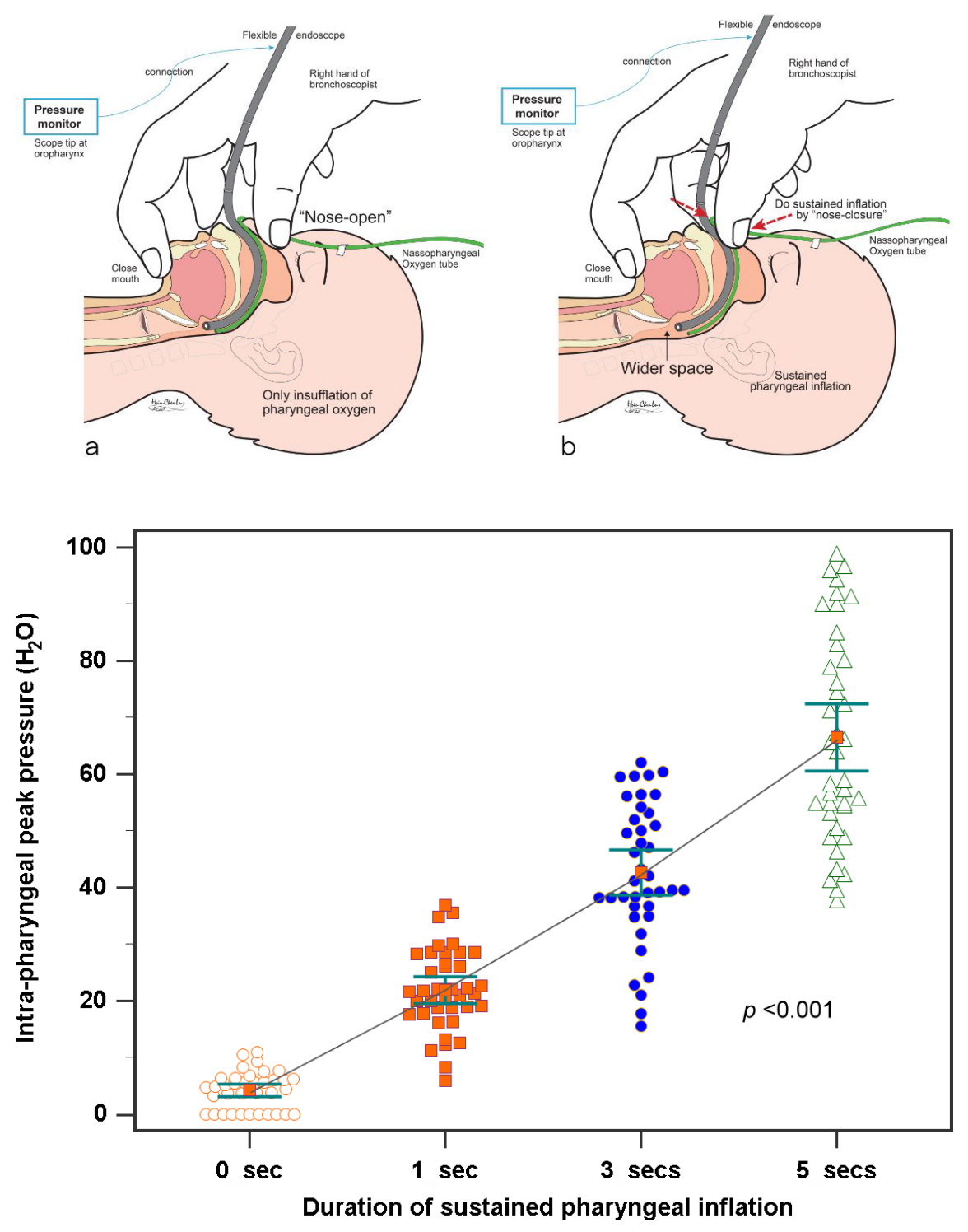


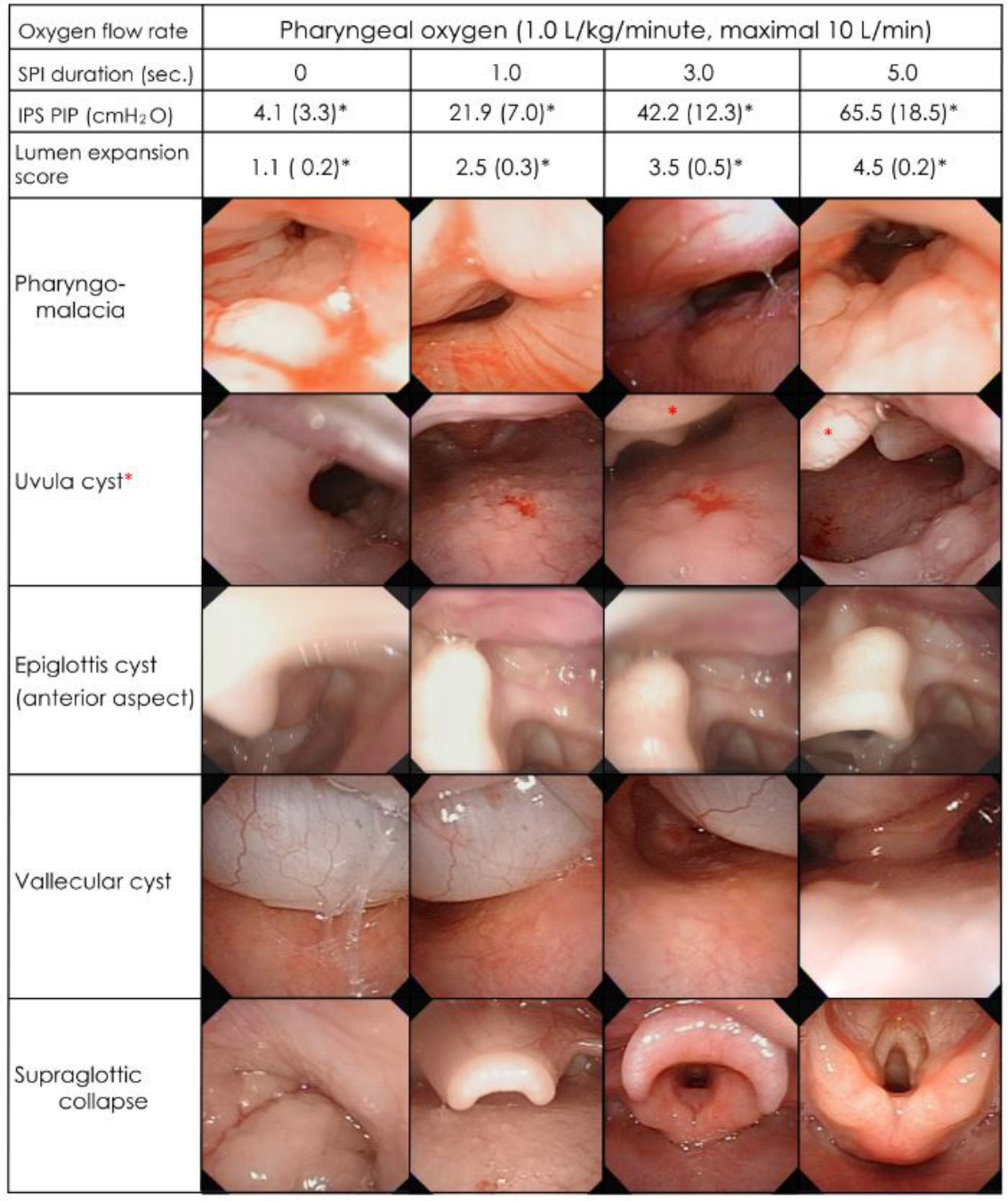

\#, images of each row take at same location of same infant. ${ }^{*}, p<0.001$. 


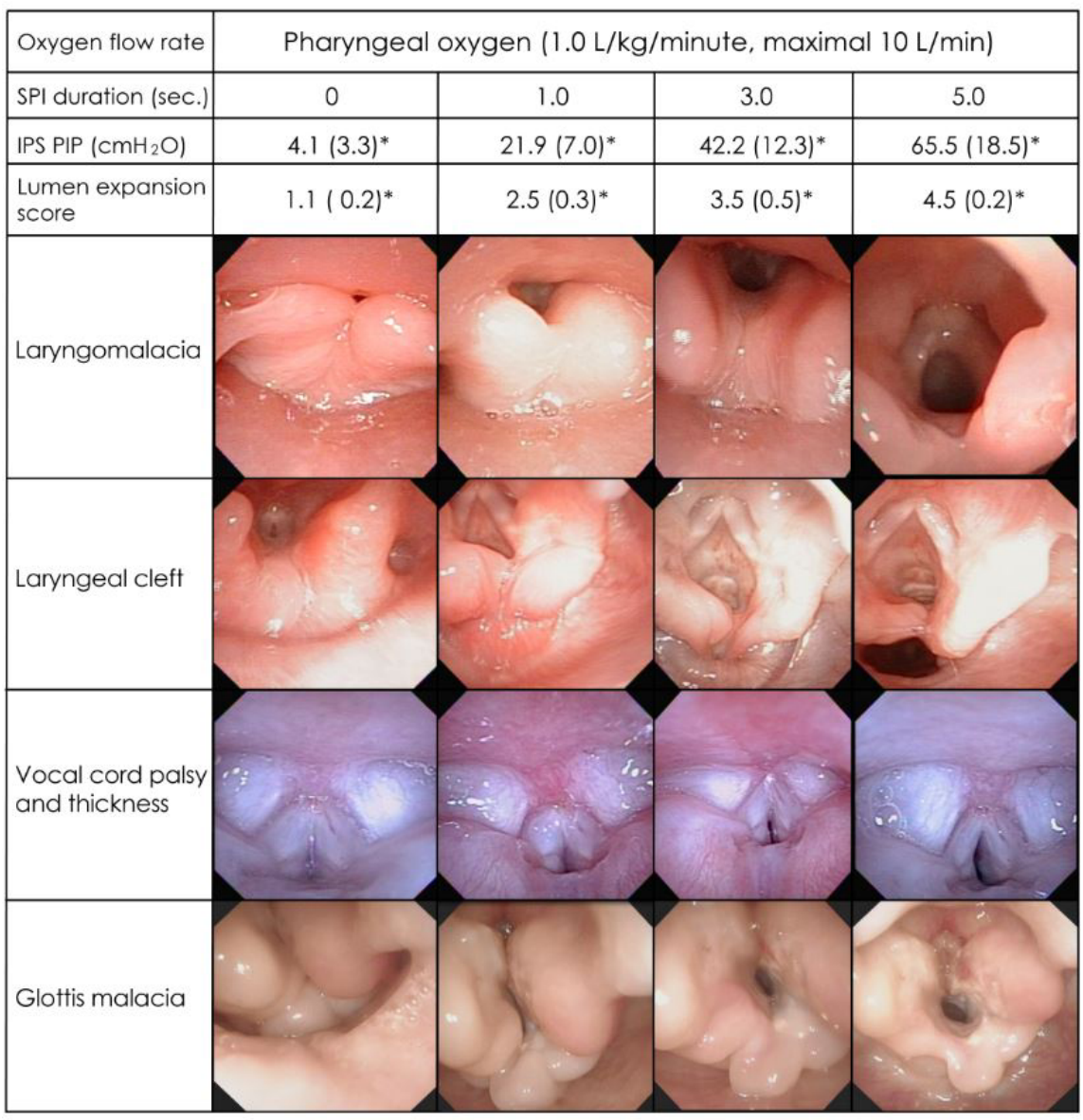

\#, images of each row take at same location of same infant. ${ }^{*}, p<0.001$. 